\title{
Editorial
}

\section{Understanding and Treating HER Better}

\author{
Marija Balic \\ Division of Oncology, Department of Internal Medicine, Medical University Graz, Graz, Austria
}

In approximately $15 \%$ of breast cancer cases, the HER2-positive subtype is diagnosed. This subtype has so far been defined by HER2 protein overexpression and/or HER2/neu gene amplification [1]. In early analyses, HER2-positivity was demonstrated to negatively impact the breast cancer prognosis, and historically, Her2-positive breast cancer was defined as the most aggressive subtype. However, the prognosis of this disease has truly been transformed. With the introduction of trastuzumab to palliative [2] and subsequently to adjuvant and neoadjuvant chemotherapy, significant clinical benefits were achieved [3, 4].

Over the last 2 decades, additional compounds have been introduced in clinical settings, and prognoses for Her2-positive patients have further improved. Increasing knowledge has led to approaches of both an escalation, as suggested in the recent Train 2 study, but also a de-escalation of treatment in the early stage, for which a greater variety is in development and will soon be revealed. By improving the treatment of early-stage Her2-positive breast cancer, relapses have become less apparent, and within the metastatic group of patients, an increase in the proportion of primary metastatic disease has been observed. Several specific challenges come hand in hand with advanced Her2-positive disease, and the most prominent problem is increasingly the occurrence and treatment of brain metastases [5]. With successful treatment, we were able to delay brain radiation and, thus, improve the quality of life of patients for a significant time, and some novel systemic therapies have shown promising re- sults effective in the central nervous system as well. Over the years, we have learned to distinguish between hormone-dependent disease within Her2-positive cases, and hormone negative, two diseases behaving in biologically and clinically different manners.

Novel drugs are entering clinics, and in this special issue, several important aspects of the treatment are covered. From the early stage and neoadjuvant and adjuvant strategies to the treatment of advanced stage, comprehensive summaries are provided.

Finally, not only systemic therapy and surgical radicality have changed dramatically over time, but also our ability to biologically characterize breast cancer and move a step closer towards personalization.

With a better understanding of biology, potential biomarkers have been recognized. And last but not least, it seems that, in the future, it may become less important to define Her2-positive disease in the standard way, by immunohistochemistry and in situ hybridization analyses. There are novel aspects of Her2 positivity, including Her2-low-positive disease and mutated breast cancers.

In this special issue of Breast Care, the spotlight is on these important aspects of HER2-positive disease. It is becoming apparent that understanding HER better increases our knowledge and impacts the development of new drugs, their implementation in clinical trials, and, finally, in the clinics. With increasing heterogeneity, design of focused and novel clinical trials is needed and will hopefully allow more precise treatment of our patients. 


\section{Conflict of Interest Statement}

The author has no conflicts of interest to declare.

\section{Author Contributions}

M.B. is the only author of this editorial.

\section{Funding Sources}

No funding was obtained for the present work.

\section{References}

1 Wolff AC, Hammond ME, Hicks DG, Dowsett M, McShane LM, Allison $\mathrm{KH}$, et al.; American Society of Clinical Oncology; College of American Pathologists. Recommendations for human epidermal growth factor receptor 2 testing in breast cancer: American Society of Clinical Oncology/College of American Pathologists clinical practice guideline update. J Clin Oncol. 2013 Nov; 31(31):3997-4013.
2 Slamon DJ, Leyland-Jones B, Shak S, Fuchs H, Paton V, Bajamonde A, et al. Use of chemotherapy plus a monoclonal antibody against HER2 for metastatic breast cancer that overexpresses HER2. N Engl J Med. 2001 Mar; 344(11):783-92.

3 Piccart-Gebhart MJ, Procter M, LeylandJones B, Goldhirsch A, Untch M, Smith I, et al.; Herceptin Adjuvant (HERA) Trial Study Team. Trastuzumab after adjuvant chemotherapy in HER2-positive breast cancer. N Engl J Med. 2005 Oct;353(16):1659-72.
4 Romond EH, Perez EA, Bryant J, Suman VJ, Geyer CE Jr, Davidson NE, et al. Trastuzumab plus adjuvant chemotherapy for operable HER2-positive breast cancer. N Engl J Med. 2005 Oct;353(16):1673-84.

5 Zimmer AS, Van Swearingen AED, Anders CK. HER2-positive breast cancer brain metastasis: A new and exciting landscape. Cancer Rep (Hoboken). 2020 Sep;e1274. https:// doi.org/10.1002/cnr2.1274 